\title{
Prognostic significance of heat shock protein 105 in lung adenocarcinoma
}

\author{
TOMOFUMI ODA ${ }^{1,2}$, EIICH MORII $^{1}$, MASAYOSHI INOUE ${ }^{2}$, JUN-ICHIRO IKEDA $^{1}$, \\ KATSUYUKI AOZASA ${ }^{1}$ and MEINOSHIN OKUMURA ${ }^{2}$ \\ Departments of ${ }^{1}$ Pathology and ${ }^{2}$ General Thoracic Surgery, Osaka University, \\ Graduate School of Medicine, 2-2 Yamada-oka, Suita, Osaka 565-0871, Japan
}

Received October 13, 2008; Accepted February 25, 2009

DOI: $10.3892 / \mathrm{mmr}+00000144$

\begin{abstract}
Heat shock protein 105 (HSP105) is overexpressed in various tumors and is known to protect cells from cytotoxic stimuli. The prognostic significance of HSP105 expression in cancer has not been fully evaluated. Here, HSP105 expression in lung adenocarcinoma was immunohistochemically examined in 116 patients: 68 males and 48 females with ages ranging from 38-81 (median 63) years. Tumor stage was I in 64, II in 16 and III in 36 patients. In non-cancerous tissues, HSP105 was expressed in the ciliated cells of bronchi. Tumor cells were constantly positive for HSP105, with varying intensities. The HSP105 score in each case was determined based on the staining intensity of the tumor cells occupying the widest area of cancerous tissue as follows: cases with tumor cells showing an intensity weaker than, comparable to and stronger than the bronchial epithelium were assigned a score of 1, 2 and 3, and numbered 84, 19 and 13 cases, respectively. HSP105 score was significantly correlated with the rate of recurrence and the stage of the disease. Univariate analysis showed that lymph node metastasis, disease stage and HSP105 score were unfavorable prognostic factors. Multivariate analysis revealed that lymph node metastasis and HSP105 score were independent prognostic factors for overall and disease-free survival. HSP105 expression is useful for the prediction of prognosis in patients with lung adenocarcinoma.
\end{abstract}

\section{Introduction}

Since 1985, lung cancer has been the most common cause of cancer-related death worldwide, with an annual worldwide mortality of 1.18 million in 2002 (1). Non-small cell lung cancer (NSCLC) comprises $75-85 \%$ of all lung cancers, with approximately two-thirds of NSCLC patients being diagnosed at an advanced stage. Despite advances in the methods of

Correspondence to: Dr Eiichi Morii, Department of Pathology, Osaka University, Graduate School of Medicine, 2-2 Yamada-oka, Suita, Osaka 565-0871, Japan

E-mail: morii@patho.med.osaka-u.ac.jp

Key words: heat shock protein 105, lung adenocarcinoma, prognosis, stress protein, immunohistochemistry, survival detection and treatment of lung cancer, the prognosis for NSCLC patients remains unfavorable. The establishment of effective therapeutic modalities is essential to improving this prognosis. Previous studies have provided information related to the factors influencing patient prognosis in NSCLC. These include clinical, pathological and molecular factors.

Heat shock protein 105 (HSP105) is a stress protein belonging to the HSP105/110 family, and plays a role in protecting cells from cytotoxic effects such as serum depletion, heat shock and the addition of hydrogen peroxide (2-6). HSP105 might contribute to the survival of cancer cells, which usually withstand cytotoxic environments such as severe hypoxia, high temperatures and the administration of doxorubicin. Recently, Hosaka et al (13) reported that the knock-down of HSP105 induces apoptosis in cancer cell lines in a caspase-dependent manner, suggesting a correlation between HSP105 expression and prognosis in cancer patients. Various types of malignant tumors, such as colorectal, pancreatic, thyroid, esophageal, breast and bladder cancers, are known to overexpress HSP107 (7). Recently, HSP105 was proposed as a useful target in cancer immunotherapy (8). Using tissue microarray, Ullmann et al evaluated the correlation of protein expression in cancer cells with the prognosis of 75 patients with lung adenocarcinoma, and found the expression of HSP together with cathepsin $\mathrm{E}$ to be prognostic markers, as revealed by univariate analysis (9). However, the staining intensity of cancer cells was arbitrarily evaluated. Here, to further evaluate the prognostic significance of HSP105 expression, immunohistochemical analysis was preformed in 116 lung adenocarcinomas. We found that the normal bronchial epithelium expressed HSP105. The staining intensity of HSP105 in cancer cells was determined by a comparison with its intensity in the bronchial epithelium, and the significance of HSP105 as an independent factor for the prediction of prognosis was evaluated by multivariate analysis.

\section{Materials and methods}

Patients. One hundred and sixteen patients who underwent surgery for lung adenocarcinoma at Osaka University Hospital between January 1993 and January 2004 were examined. Histological stage was determined according to the 6th edition of the Union International Contre le Cancer - TNM staging system (10). Histologic specimens were fixed in $10 \%$ formalin 

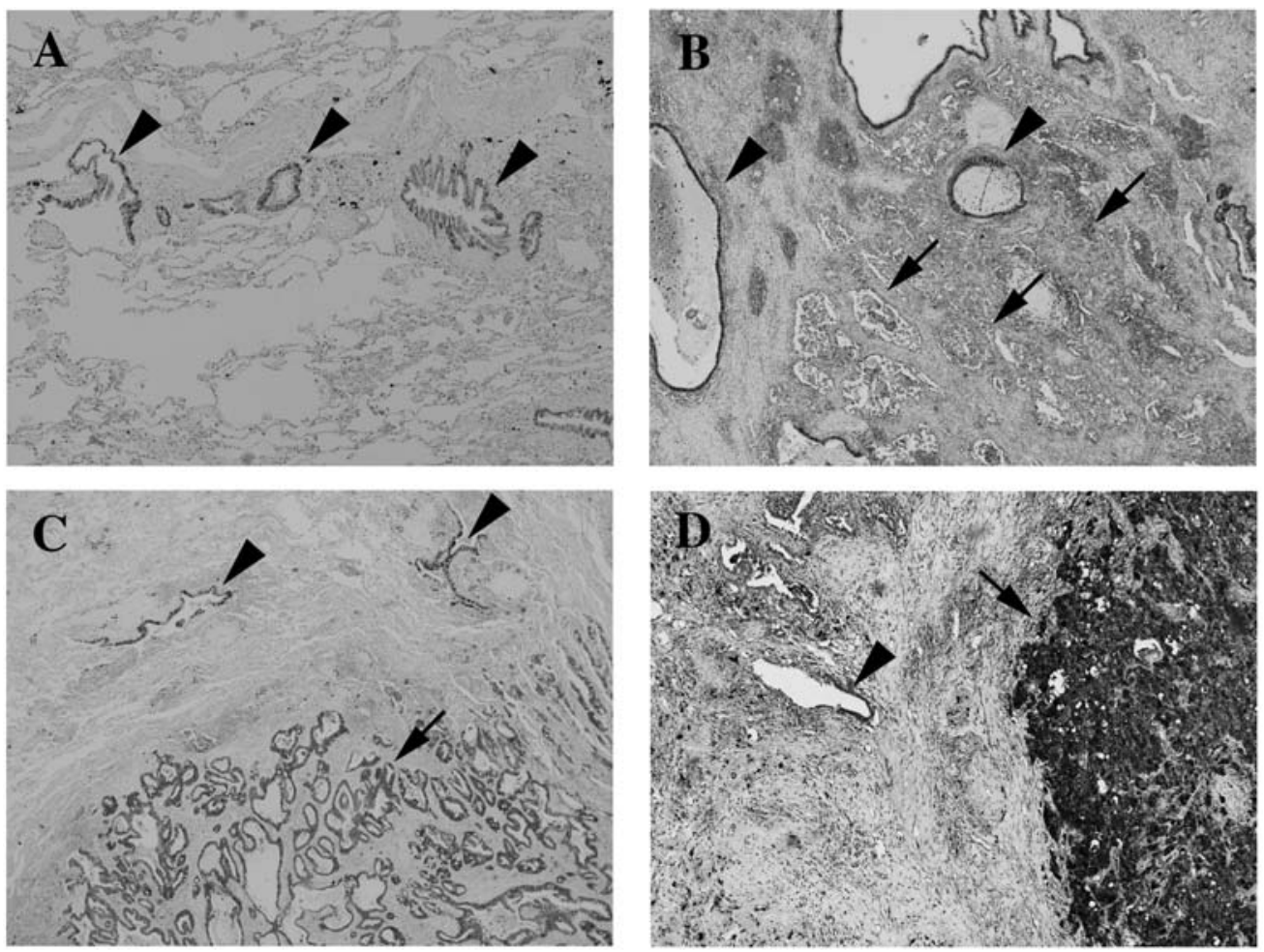

Figure 1. HSP105 expression in lung adenocarcinoma. (A) HSP105 expression was found in the bronchial epithelium (arrowheads) but not in the alveolar epithelium of non-cancerous tissues. (B-D) Tumor cells expressed HSP105 with varying intensities. Cases with more than half their tumor cells (arrows) showing weaker (B), equal (C) and stronger (D) HSP105 staining than in the bronchial epithelium were assigned a score of 1, 2 or 3, respectively. Avidin-biotinperoxidase complex method; $\mathrm{x} 100$.

Table I. Characteristics of the 116 patients with pulmonary adenocarcinoma.

\begin{tabular}{lc}
\hline & No. of patients \\
\hline Gender & 68 \\
Male & 48 \\
Female & \\
T category & 54 \\
1 & 43 \\
2 & 11 \\
3 & 8 \\
4 & \\
N category & 78 \\
N0 & 7 \\
N1 & 27 \\
N2 & 4 \\
N3 & \\
Stage of disease & 64 \\
I & 16 \\
II & 36 \\
III & \\
Recurrence & 55 \\
Yes & 61 \\
No & \\
Prognosis & 35 \\
Deceased & 17 \\
Alive (with recurrence) & 64 \\
Alive (with no recurrence) & \\
\hline
\end{tabular}

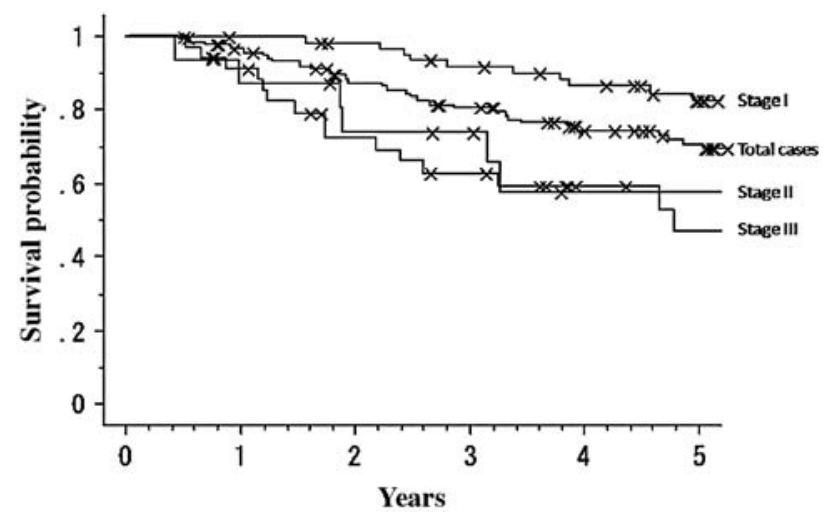

Figure 2. Overall survival (OS) in the 116 lung adenocarcinoma cases. OS in all patients and in patients with stage I, II and III tumors was 69.5, 82.6, 57.6 and $47.3 \%$, respectively.

and routinely processed for paraffin embedding. Paraffinembedded specimens were stored in a dark room in the Department of Pathology of Osaka University Hospital at room temperature and were sectioned at a thickness of 4-mm at the time of staining. All patients were followed up with laboratory examinations including routine peripheral blood cell counts at 1- to 6-month intervals, chest roentgenograms, computed tomographic scans of the chest and endoscopic examinations of the bronchus at 6- to 12-month intervals. The follow-up period for survivors ranged from 5 to 150 (median 61) months. The study was approved by the Ethics Review Board of the Graduate School of Medicine, Osaka University. 
A
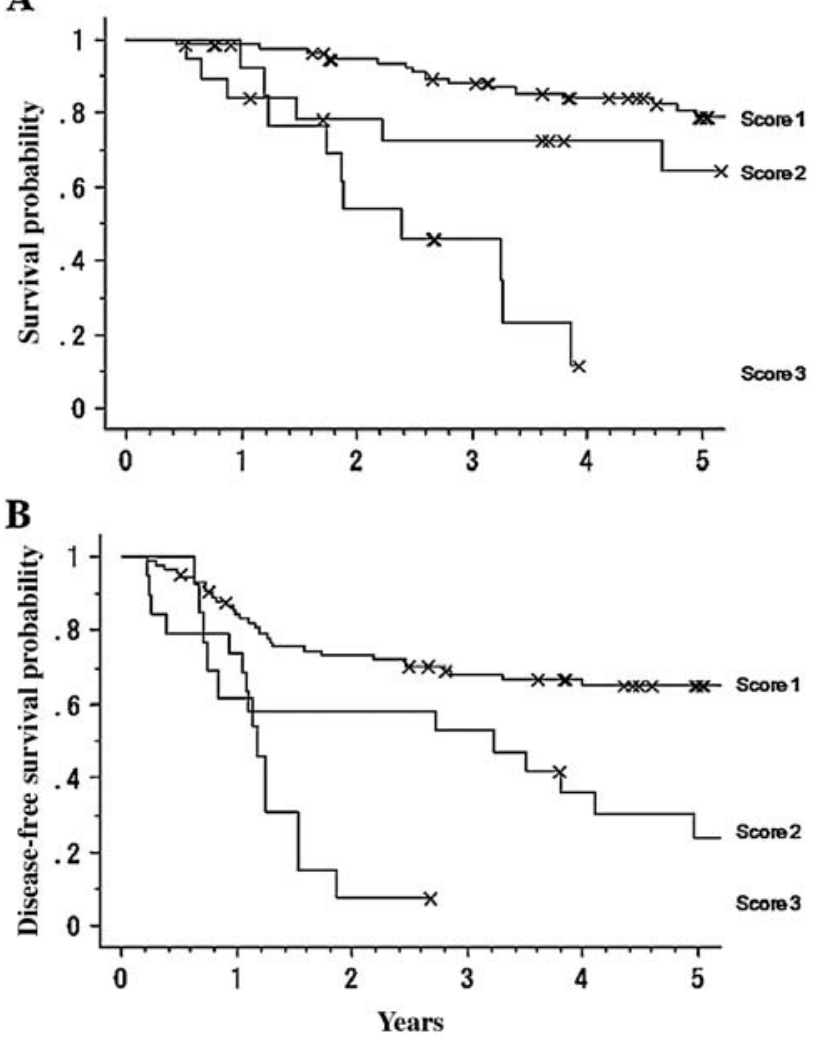

Figure 3. Overall survival (A) and disease-free survival (B) curves in cases with HSP105 scores of 1, 2 and 3. (A) The P-value between score 1 and 2, score 2 and 3 , and score 1 and 3 was $0.078,0.016$ and $<0.0001$, respectively. (B) The P-value between score 1 and 2 , score 2 and 3 , and score 1 and 3 was $0.022,0.032$ and $<0.0001$, respectively.

Immunohistochemistry. An immunoperoxidase procedure was performed on the paraffin-embedded sections using the standard avidin-biotin-peroxidase complex method. After antigen retrieval with a Pascal pressurized heating chamber (Dako A/S, Glostrup, Denmark), the sections were incubated with anti-HSP105 (Novocastra, Newcastle, UK; dilution; 1:100), anti-active caspase 3 (Abcam Ltd., Cambridge, UK; 1:100) or anti-Ki-67 antibody (Dako; 1:100). As the negative control, staining was carried out in the absence of primary antibody. Staining of the bronchial epithelium was used as the internal positive control for HSP105. The HSP105 score in each case was determined based on the staining intensity of the tumor cells occupying the widest area of cancerous tissue as follows: cases with tumor cells showing an intensity weaker than, comparable to, and stronger than the bronchial epithelium were assigned a score of 1,2 and 3, respectively (Fig. 1A-D). The apoptosis index was determined by evaluating the proportion of active caspase 3-positive tumor cells. Cases in which $>3 \%$ of tumor cells were active caspase 3-positive were categorized as having a high apoptosis index. Proliferation of tumor cells was evaluated by Ki-67 labeling. Cases in which $>3 \%$ of tumor cells were Ki-67positive were categorized as having a high proliferation index. Immunohistochemically-stained sections were evaluated independently by two investigators (T.O. and E.M). For patients with discrepant findings, the results were compared and discussed.
Table II. Correlation of heat shock protein 105 expression in lung adenocarcinoma with clinicopathological factors.

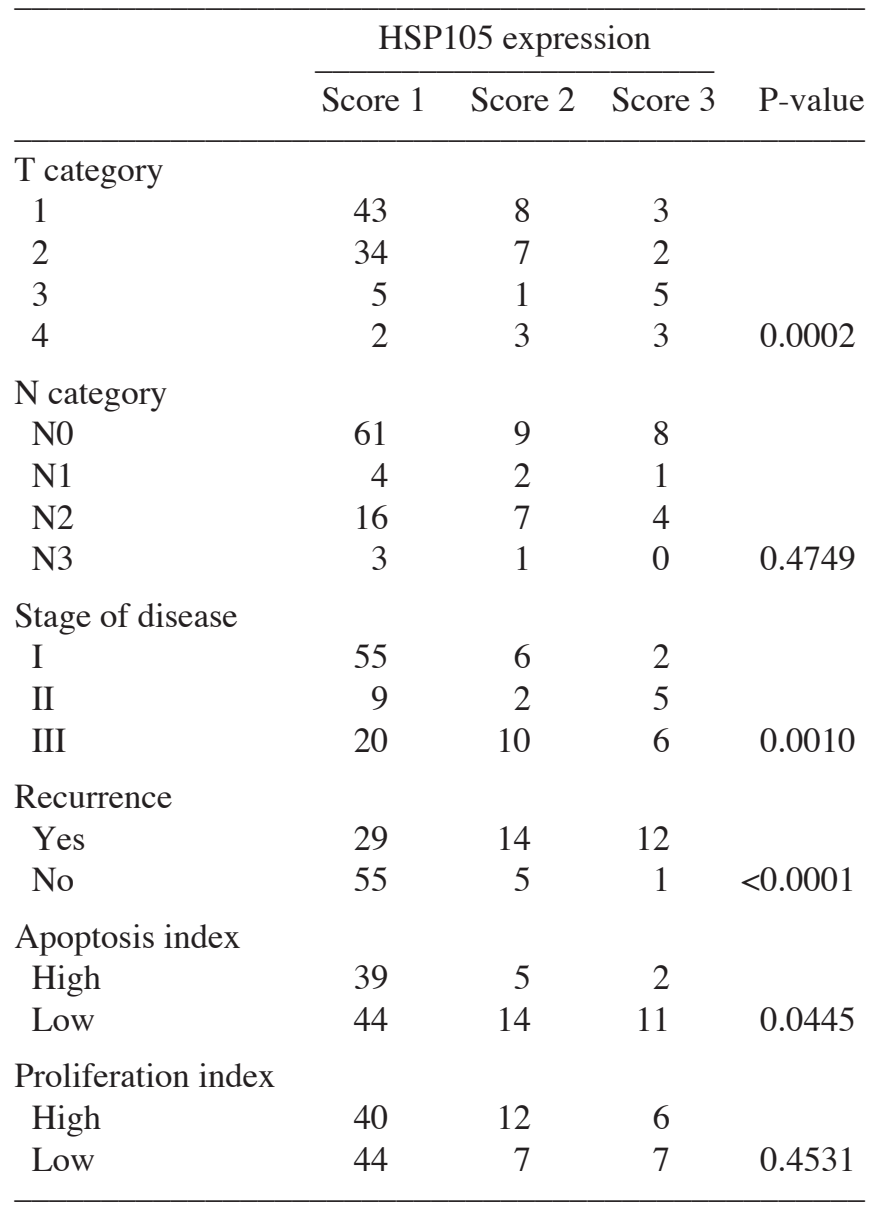

Statistical analysis. All statistical analysis was performed using StatView software (SAS Institute Inc., Cary, NC). Five-year overall survival (OS) and disease-free survival (DFS) were calculated by the Kaplan-Meier method, and differences in survival curves were analyzed by the log-rank test. Independent prognostic factors were analyzed by the Cox's proportional hazard regression model. The presence of a statistically significant difference was denoted by $\mathrm{P}<0.05$.

\section{Results}

Clinical findings. The clinical findings are summarized in Table I. There were 68 males and 48 females with ages ranging from 38 to 81 (median 63) years. The size of the main tumor ranged from 8 to 70 (median 30.5 ) $\mathrm{mm}$. Tumor stage was I in 64 , II in 16 and III in 36 patients. Tumor recurrence was found in 55 cases, with the interval from surgery ranging from 2.5 to 78.7 (median 13.2) months. Five-year OS in all patients and in patients with stage I, II and III tumors was 69.5, 82.6, 57.6 and 47.3\%, respectively (Fig. 2). DFS in all patients and in patients with stage I, II and III tumors was 51.5, 80.9, 59.1 and 36.5\%, respectively (Fig. 3A and B).

Expression of HSP105 and its correlation with clinical factors. With immunohistochemistry, positive staining was observed in the ciliated cells of bronchi and macrophages in the alveolar 


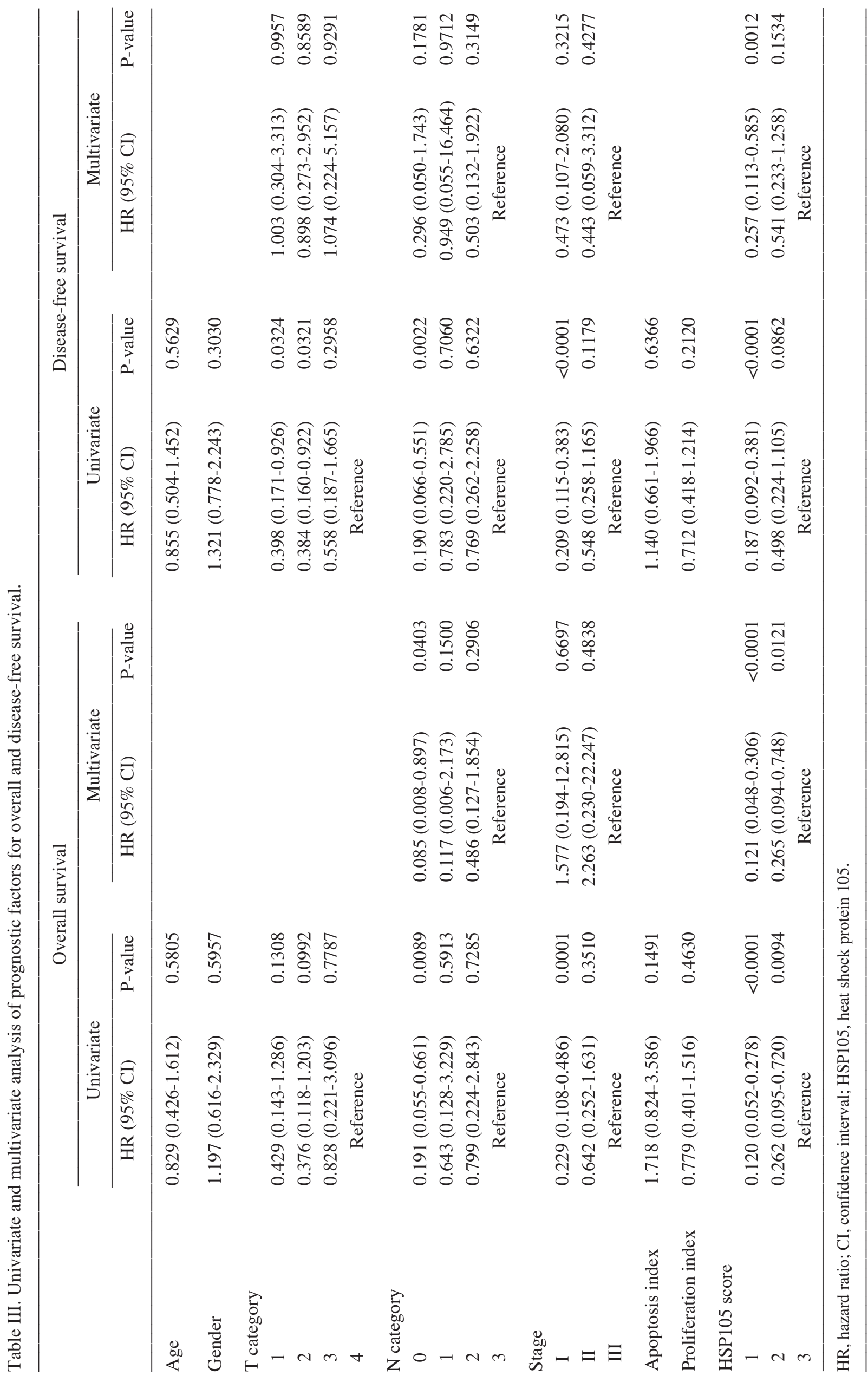


wall and lumen, but not in alveolar epithelial cells in noncarcinomatous areas (Fig. 1A). Tumor cells were constantly positive for HSP105, but with varying expression levels. The HSP105 expression level in each case was classified based on the staining intensity of the tumor cells occupying the widest area of cancerous tissue as follows: cases with tumor cells showing an intensity weaker than, comparable to, and stronger than the bronchial epithelium were assigned a score of 1,2 and 3, respectively (Fig. 1A-D). Among 116 patients, 84 were assigned a score of 1,19 a score of 2 and 13 a score of 3 .

The correlation of HSP105 score with clinical features is shown in Table II. $\chi^{2}$ analysis revealed $\mathrm{T}$ category, disease stage, tumor recurrence and a low apoptosis index to be significantly correlated with a high HSP105 score. Patients with score 1 and score 2 tumors had a significantly improved OS than those with score 3 tumors $(\mathrm{P}<0.0001$ and $\mathrm{P}=0.016$, respectively) (Fig. 3A). Patients with score 1 and score 2 tumors had a significantly longer DFS than those with score 3 tumors $(\mathrm{P}<0.0001$ and $\mathrm{P}=0.032$, respectively) (Fig. 3B).

Univariate analysis revealed lymph node metastasis, advanced stage and high HSP105 score to be significant unfavorable factors for both 5-year OS and DFS (Table III). Multivariate analysis revealed that lymph node metastasis and HSP105 score were independent prognostic factors of both 5-year OS and DFS (Table III).

\section{Discussion}

Patient characteristics such as gender, age and 5-year OS were similar in the present study to those of previous reports (11). Moreover, the results of univariate and multivariate analysis regarding the prognostic significance of clinicopathological factors, such as lymph node metastasis and stage of disease, were also in accordance with previous studies. These findings indicate that the results obtained from the present cases are commonly applicable.

Among the clinicopathological factors examined, high HSP105 expression was related to advanced disease stage, high $\mathrm{T}$ category, a low apoptosis index and a high probability of recurrence. A low apoptosis rate may explain the larger size and higher recurrence rate of tumors with a high HSP105 score compared to those with a low score. Since HSP105 expression was not correlated with N category, HSP105 might not affect the metastatic potential of lung adenocarcinoma.

The present findings in 116 cases with lung adenocarcinoma, that patients with score 1 and score 2 tumors exhibit significantly longer OS and DFS than those with score 3 tumors, are in agreement with those reported by Ullmann et al (9), who found survival time to be longer in patients with low HSP105 expression in a study of 75 cases. Using multivariate analysis, we further demonstrated that HSP105 expression is an independent prognostic factor for OS and DFS.

The neuronal cell line PC12 stably overexpressing HSP105 exhibited resistance to caspase-mediated apoptosis (12). In addition, the knock-down expression of HSP105 induced apoptosis in a caspase 3-dependent manner in the colon cancer cell line HCT116. Consistent with these reports, high expression of HSP105 was inversely correlated with active caspase 3 positivity in clinical samples of lung adenocarcinoma. These findings indicate that the caspase-mediated apoptosis of tumor cells could be suppressed by the overexpression of HSP105. This might explain why the prognosis for patients having tumors with a high HSP105 score is less favorable than that of patients having tumors with a low score.

According to studies by Hatayama et al (12) and Hosaka et al (13), overexpression of HSP105 did not affect the growth rate of the neuronal cell line PC12 and fibroblast cell line $\mathrm{NIH} / 3 \mathrm{~T} 3$. Indeed, no correlation was found between HSP105 expression and the proliferation index in the clinical samples of the present study. Rather than increasing proliferative activity, HSP105 appeared to exert an anti-apoptotic effect.

Although the overexpression of HSP105 has been demonstrated in various types of tumors, the significance of HSP105 as a prognostic factor has only been studied in lung adenocarcinoma. Further studies are necessary to determine whether HSP105 expression is a useful marker for the prediction of prognosis in other types of cancer.

\section{Acknowledgements}

The authors thank Ms. Megumi Sugano, Ms. Etsuko Maeno and Ms. Takako Sawamura for technical assistance. This work was supported by grants from the Ministry of Education, Culture, Sports, Science and Technology of Japan.

\section{References}

1. Parkin DM, Bray F, Ferlay J and Pisani P: Global Cancer Statistics, 2002. CA Cancer J Clin 55: 74-108, 2005.

2. Hatayama T, Honda K and Yukioka M: HeLa cells synthesize a specific heat shock protein upon exposure to heat shock at $42^{\circ} \mathrm{C}$ but not at $45^{\circ} \mathrm{C}$. Biochem Biophys Res Commun 137: 957-963, 1986.

3. Yasuda K, Nakai A, Hatayama T and Nagata K: Cloning and expression of murine high molecular mass heat shock protein, HSP105. J Biol Chem 270: 29718-29723, 1995.

4. Ishihara K, Yasuda $\mathrm{K}$ and Hatayama T: Molecular cloning, expression and localization of human $105 \mathrm{kDa}$ heat shock protein, hsp105. Biochim Biophys Acta 1444: 138-142, 1999.

5. Yamagishi N, Nishihori H, Ishihara K, Ohtsuka K and Hatayama T: Modulation of the chaperone activities of Hsc70/ Hsp40 by Hsp105alpha and Hsp105beta. Biochem Biophys Res Commun 272: 850-855, 2000.

6. Easton DP, Kaneko Y and Subjeck JR: The hsp110 and Grp170 stress proteins: newly recognized relatives of the Hsp70s. Cell Stress Chaperones 5: 276-290, 2000.

7. Kai M, Nakatsuka T, Egami H, Senju S, Nishimura Y and Ogawa M: Heat shock protein 105 is overexpressed in a variety of human tumors. Oncol Rep 10: 1777-1782, 2003.

8. Yokomine K, Nakatsura T, Minohara M, Kira J, Kubo T, Sasaki Y and Nishimura Y: Immunization with heat shock protein 105pulsed dendritic cells leads to tumor rejection in mice. Biochem Biophys Res Commun 343: 269-278, 2006.

9. Ullmann R, Morbini P, Halbwedl I, Bongiovanni M, GoggKammerer M, Papotti M, Gabor S, Renner H and Popper HH: Protein expression profiles in adenocarcinomas and squamous cell carcinomas of the lung generated using tissue microarrays. J Pathol 203: 798-807, 2004.

10. Sobin LH and Wittekind CH (eds): TNM Classification of Malignant Tumors. 5th edition, John Wiley \& Sons, Inc., New York, 1997.

11. Suzuki K, Nagai K, Yoshida J, Nishimura M, Takahashi K, Yokose $\mathrm{T}$ and Nishiwaki Y: Conventional clinicopathologic prognostic factors in surgically resected nonsmall cell lung carcinoma. Cancer 86: 1976-1984, 1999.

12. Hatayama T, Yamagishi N, Minobe E and Sakai K: Role of hsp105 in protection against stress-induced apoptosis in neuronal PC12 cells. Biochem Biophys Res Commun 288: 528-534, 2001.

13. Hosaka S, Nakatsuka N, Tsukamoto H, Hatayama T, Baba H and Nishimura Y: Synthetic small interfering RNA targeting heat shock protein 105 induces apoptosis of various cancer cells both in vitro and in vivo. Cancer Sci 97: 623-632, 2006. 